\title{
Peter Morris
}

The job title in the biographical section of this Obituary (BMJ 2016;354:i4870, doi:10.1136/bmj.i4870) is incorrect. It should read consultant anaesthetist. We apologise for this error. 